soon after which she had a fearful gush of hæmorrhage. She was put into bed and plugged. She was delivered of a still-born child after four hours ; but she never rallied, dying in two hours afterwards, having had no more hxmorrhage.

Concealed Acidental Himorrhage.-Case I, aged 38 ; ninth pregnancy ; had a fall two months before, which brought on hæmorrhage, from which with care she recovered, and went on to the full term. The child was born after six hours' labour, and she died in twenty-seven hours from syncope. - Case II, aged 28 ; fourth pregnancy; was seized with faintness and vomiting; feeble pulse and great prostration; she was well bound up and supported with stimulants until pains came on ; after the expulsion of the child a large mass of clots followed. She was very feeble and suffered from intense nervous excitability for eighteen days, when she died after two days' pneumonia.

Spasmodic Asthma.-Aged 37 ; tenth pregnancy; was thrown out of a trap at about the sixth month, which shook her much, and from which she had slight concussion. The attack of asthma came on three days before labour; she had albuminous urine; died on the eleventh day.

Bronchitis. - Aged 34; premature labour on account of the bronchitis; she died in two days.

Pneumonia.-Aged 43 ; thirteenth pregnancy. This woman got out of bed on the night of the fourth day after delivery, and, without shoes or stockings, or any covering other than her night-dress, traversed a landing to the rooms of her children to see if they were safe. She was seized with pneumonia, and died on the ninth day.

Although the proportion of deaths has been I in I25, it is singular that I attended 250 labours before meeting with a fatal case; and, as if to prove to me the fallacy of calculating statistically from a small number of data, I found the next three deaths to occur in a much more rapid sequence-I in 66.3-although I subsequently had an interval, in which 45 I cases were attended without meeting with a death. I should also add that, after attending the first fatal case of puerperal fever, in which I made a post mortem examination, I delegated my midwifery cases to a brother practitioner for the space of one month; and whenever I possibly could, which was nearly always, I got cases of erysipelas attended by proxy also.

The ages of the patients and the duration of labour have been omitted from this account, as in many of the cases reliable information could not be satisfactorily obtained, whilst some facts and data relating to the duration of pregnancy I have reserved for a future occasion.

\section{CHLOROFORM ACCIDENTS.}

\section{BY J. T. CLOVER, F.R.C.S.}

THE attention of medical men has been recently called to this subject, by a revision of Professor Lister's article on anæsthetics in Holme's System of Surgiry. If I understand Mr. Lister, he maintains-I. That in deaths from chloroform the breathing fails before the circulation ; 2 . That the chief danger arises from laryngeal obstruction ; 3 . That the chief duty of the administrator is to watch for laryngeal obstruction, and draw out the tongue with forceps when it occurs; 4 . That it is useless to watch the pulse; 5 . That chloroform may be given as safely by means of a towel as by any apparatus. My experience obliges me to differ from Mr. Lister on these points. I have administered chloroform more than seven thousand times, and ether, tetrachloride of carbon, ethylidenchlorid, the compound "bichloride of methylene", and nitrous oxide, in four thousand other cases. I have never drawn out the tongue, and never lost a patient from any anæsthetic. My aim has always been to give the anxsthetic equally mixed, and in the smallest proportion needed for the purpose, so that, if the effect became greater than I wanted, there might be as little of the agent as possible in the chest requiring to be got rid of.

For several years I gave chloroform upon lint held at a distance from the face, but covered over with a towel; but I found it impossible always to prevent the patient from inhaling more than I desired. This induced me to contrive an apparatus which I exhibited in 1862 , and have used constantly since. It enables me to administer the vapour of three minims and a quarter of chloroform with every one hundred cubic inches of air. This proportion rarely excites coughing or swallowing ; and whenever any laryngeal obstruction has been threatened I have prevented it by raising the chin, and never have been obliged to draw out the tongue.

It is my habit when giving anæesthetics to watch the pulse as well as the breathing, and $\mathrm{I} \mathrm{am}$, therefore, better able to speak of the effect of chloroform upon the heart than those who disregard the pulse. It appears that in Mr. Lister's practice the necessity for dragging forward the tongue is of frequent occurrence. Either his method of giving chlo- roform is not the best that may be devised for preventing the choking, or else the severe process of seizing the tongue with artery-forceps when the choking occurs is not so imperative as he supposes. Probably some of his patients were so affected by the pungency of the vapour that the simpler expedient of raising the chin well away from the sternum, which I have never found to fail, might have been sufficient.

There is an obstruction to breathing from closure of the glottis of a reflex character. It is often produced when a ligature is applied to a hæmorrhoid or to the pedicle of an ovarian tumour ; but the obstruction Mr. Lister dwells upon is, I suspect, nothing more than an act of swallowing imperfectly performed. Every one must have noticed that swallowing is very commonly excited by the vapour of chloroform when first inhaled, and the stronger the vapour, the more frequent and annoying to the patient it becomes. There is reason to think that, under chloroform, the co-ordination of the movements which constitute deglutition is in. terfered with, and that in consequence it lingers in the second stage, when, as we know, there is normally a stoppage to the passage of air.

It is a remarkable fact that we do not get the laryngeal stoppage from nitrous oxide, although it produces a very deep stertor. In some thousands of cases I have continued to give the gas until this stertor was produced, but I do not remember a single case where the larynx was completely stopped. It is probable that the immunity from this accident depends upon the circumstance that the gas is so bland that it never stimulates the throat enough to produce the act of swallowing.

In giving chloroform I have met with several cases of partial obstruction of the larynx, where the mere depressing or raising of the chin was enough to close or open the passage. In such cases, if I had tried to open the mouth by pushing down the chin, I should by that very means have caused total obstruction, such as Mr. Lister describes; and then, no doubt, dragging out the tongue would have been followed by a free inspiration, and probably louder and freer than the one produced by the simple process of elevating the chin.

I am convinced that the chief cause of danger is the effect of chloroform upon the heart. Several deaths have occurred where failure of the pulse was noticed before the breathing became difficult; these cases should have recovered if Mr. Lister's theory be correct. Supposing the larynx to be obstructed at a time when the heart is acting well, and when not more than four or five per cent. of chloroform vapour is in the chest, the narcotism will diminish for the following reason. The blood circulating through the general system deposits a part of the chloroform in the tissues, and, passing through the lungs, it soon reduces the proportion of chloroform there present, and is no longer supplied with a sufficient quantity to keep the nervous centres under its full influence.

Those who have had experience in giving chloroform in a manner to secure that the air breathed only contains four per cent., must have noticed that patients who strain and hold their breath for half a minute or more, as they fell into a state of insensibility, are less under the influence of the aniesthetic when they recommence breathing than when they began to hold their breath. Supposing, however, that the lungs contained ten or twelve per cent. of chloroform, a very different result would occur : the narcotism would increase, for the heart's action would first be diminished, and as a consequence of this the blood would pass through the lungs more slowly, and by passing more slowly would have time to take up still more of the chloroform, and have a dangerous or fatal effect upon the ganglia of the heart. This is the worst form of accident that can happen; for it is obvious that, without effective circulation through the lungs, drawing out the tongue and artificial respiration would be useless.

The pulse varies considerably under the influence of chloroform; it often fails and is scarcely perceptible when sickness is about to take place ; also when extensive incisions are made, or important nerves are injured. Whenever chloroform is given stronger than four and a half per cent., the pulse loses force rapidly. Experiments with the hrmadynamometer show that the arterial pressure diminishes when animals are kept under the influence of chloroform. If chloroform vapour is administered very strongly through a trachea-tube, it stops all action of the heart in sixteen seconds. It cannot be too strongly impressed on those who administer chloroform that the same proportion which would be safe when the blood passes quickly through the lungs, might be dangerous if the blood moved slowly, because in the latter case it would take up more chloroform.

I will mention a single case out of many which tend to show both that the pulse must be watched, and that no higher proportion of the anæsthetic should be given than is absolutely needful. A gentleman, fifty years of age, was inhaling chloroform freely, when, finding that
his pulse became unsteady and soft, I discontinued the chloroform whilst he was still breathing satisfactorily. I felt the pulse become less and less, and in a few seconds cease; as he continued to breathe freely 
and was recumbent, I merely waited, hoping that the pulse would return ; and I saw his respirations gradually become fainter and at longer intervals, till at the end of a minute or so they also ceased, and he seemed dead. I now kept up the movements of the chest artificially for about a minute, when he breathed again spontaneously, and his pulse returned. In a few minutes he entirely recovered. It was the pulse which gave me the first warning of danger. Although I withdrew the chloroform whilst he was still breathing, he had a narrow escape, and I cannot avoid the conclusion that had he inhaled chloroform air instead of pure air, during the minute which followed the stopping of his pulse, he must have died.

\section{ON THE CONTAGION OF SCARLATINA AND SMALL-POX.}

By T. T. PYLE, M.D.,

Physician to the Sunderland Infirmary, etc.

AT the present moment, when the public mind is agitated by doubts as to the necessity for vaccination on the one side, and by the fear of catching small-pox on the other, it may not be amiss to bring forward a few simple facts illustrative of the manner in which contagious diseases are spread abroad, and indicative of the means by which they may be prevented by timely care and attention. I maintain that it is by the neglect of a few very simple rules that our population is decimated by these infectious diseases; and we shall never check their progress until medical attendants and the heads of families are careful in carrying out a system of isolation on the appearance of fever in their villages or in their houses, and also in carefully tracing the virus-bearer so as to catch and imprison him until he is thoroughly disinfected and has ceased to be a pest to society. For these reasons, I call attention to this important subject, and venture to relate the following cases which have come under my observation in my own family and practice.

I shall begin by a case of scarlet fever that took place in my own house in the latter part of 1870 . My eldest child, Ethel, aged 8 years, went to play with her little cousins for two days consecutively, and, on the evening of the second day, she awoke from her sleep with violent voniting, and complained of pain in her throat. Two days after this, the eruption of scarlatina appeared. She had a rather severe attack; but, I am thankful to sav, passed through the disease without any very formidable symptoms. It appeared that the housemaid at her cousins' house, about a week or so before, had suffered from sore-throat and excessive vomiting and prostration: she had been confined to bed for a couple of days, but was afterwards able to go about the house and perform her duties, though extremely weak. I made every inquiry where the child could have caught the infection. I had myself not attended any case of scarlatina for several months before that period. The laundress had not washed any clothes except for our family and the family of a patient of my own, whose children were quite healthy; and the only conclusion that could be arrived at was, that this housemaid had had a slight attack of scarlatina, and gave it to my little girl, who, perhaps, was rather in a condition to receive it, having just been convalescent from an attack of bronchitis.

The second case I can trace very clearly. Miss M., a governess from a school in Sheffield, came to Sunderland for the Christmas holidays, and consulted me for a chest-affection. On her next visit to me, about ten days afterwards, she was very depressed, and gave the following reason. Before leaving the school at Sheffield, a favourite pupil took scarlet fever; she nursed the child throughout its illness till its death. A few days after her return home, she wore the same dress that she had while she attended on her pupil. One of her little brothers took scarlet fever, and died within a few days. Three or four of the other children in the house also took the disease, but she herself never had the least symptom of it.

The third case occurred at a colliery village some few miles from this town, and one over which I have the medical supervision. The resident medical gentleman informed me that there had not been a case of scarlatina in the place for more than a year before. The villacre contains somewhere between three and four thousand inhabitants. When I was first called to see the child, the rash of scarlet fever was well developed, and the parents could give me no idea how the disease could have been caught. The drainage was excellent, the children were well fed, and the air good, being on the sea-coast. This child ran through the course of the disease in a mild form, and recovered. By very careful inquiry, I discovered that a relative of the parents had sat up all night in Sunderland a few days before this period, nursing a neighbour's child that had been ill and delirious, but, as they stated, merely with a very bad sore throat. Before leaving this case, I ought to state that I took every precaution to prevent the spreading of the disease in the village. In spite of my efforts, some of the friends visited the house, and the fever appeared in two other houses in the place, in one of which three cases of scarlatina occurred, and in the other house one case. By impressing on these people the great impertance of preventing any communication whatever with their friends, I was gratified to find that my instructions were fully carried out; and no other cases broke out except in the house where the first case was discovered. A brother of the child first affected was not at home when the fever appeared, and I ordered that he should be kept away for some time. After seven weeks, and when the child affected had become convalescent, the other child was brought home. I could not clearly ascertain if it had worn any of the clothing of the affected child; but, a few days after its return, it had a mild but distinctive attack of scarlet fever. I am happy to state that since that time, now several weeks ago, no fresh case of scarlatina has occurred in the village.

The following case will illustrate the direct contagion of small-pox, which is now occupying so much attention. It occurred at the same colliery village, and was brought from Hartlepool (a town distant about twenty miles) by a man in the desquamative stage, or, as they expressed it, "red raw", after the disease. He was invited to the house for change of air, and stayed about a week. Two days after he left, one of the children who had never been vaccinated, and whom the man had fondled a great deal and frequently had to play with him, took the confluent form of small-pox. The other children in the house had also not been vaccinated, but the Resident Surgeon and Public Vaccinator for the district at once had them vaccinated. They did not take the disease; and, by a proper surveillance of the house, and preventing any communication with it, no other cases have yet appeared.

\section{CLINICAL ILLUSTRATIONS OF CUTANEOUS DISEASE.}

By ERASMUS WILSON, F.R.S.,

Professor of Dermatology in the Royal College of Surgeon.

Melasma Frontis, with General Melasma in Putches, orivinatinr in Cachexia.-March 27 th, 187 I. A robust woman, aged 40 , is troubled with a greyish-black stain which covers all that portion of the forehead which is exposed to the light. Her neck is also swarthy, and the integument of the whole body is darkened with pigmentary blotches of irregular figure and extent. This melasmic affection has been two years in progress, and is now somewhat less than it was some months back. Her health has been much shaken by numerous causes of exhaustion. Some years ago, she had a severe attack of rheumatic gout ; this was followed by several miscarriages; and, immediately before the darkening of the skin was first observed, she suffered very considerably from abdominal spasm, accompanied with flatulence.

Perverted pigmentation of the skin in the form of melasma may always be traced to causes affecting innervation, assimilation, and sanguification. Such causes existed in the present instance; and the pigment-substance would seem to owe its origin to the destruction of the red corpuscles of the blood, for anæmia, in a greater or less degree, is always present with melasma; and in the present instance the sclerotic was anæmically pale.

Erythema Solare, affecting the Face and Hands on Expositie to the Ray's of the Sun.-April 4th, I87 I. Pellagra, the mal del soli, a disease of the South of Europe, has made us acquainted with an erythema due to a neuropathic condition of the skin; the disease subsequently invading the whole of the nervous system and brain, and ending in madness. Such a type of disease as this is necessarily recalled to the mind when anything of a similar nature, however remote, is brought under our notice; and it is as a far distant resemblance of the earliest stage of this affection that I now record the following case.

A healthy-looking young woman, aged 38 , complains of the scorching effect of the sun upon her face and hands; and, to illustrate her complaint, she shows me the back of her hand, upon which she has allowed the light of an early April sun to impinge while awaiting her turn of consultation, about an hour. Sure enough, the hand presents a well marked erythema of a dull red or purplish-red colour-a slight degree, she observes, of the appearance produced during the warmer months of the vear. The inference is evident. Her skin must be unusually and morbidly sensitive to be injured to such an extent by the actinic rays of the sun. She dates back this hypersensitiveness of the skin to a period of seven years; and since that time she has also noticed that her skin is unusually dry and lacking of perspiration.

Prarigo Mitis, from imperfect Assimilation. - Narch IIth, I87I. A 